\title{
Mathematical modelling of the automated FADU assay for the quantification of DNA strand breaks and their repair in human peripheral mononuclear blood cells
}

Michael Junk', Judy Salzwedel${ }^{2}$, Thilo Sindlinger ${ }^{2}$, Alexander Bürkle ${ }^{2}$ and Maria Moreno-Villanueva2*

\begin{abstract}
Background: Cells continuously undergo DNA damage from exogenous agents like irradiation or genotoxic chemicals or from endogenous radicals produced by normal cellular metabolic activities. DNA strand breaks are one of the most common genotoxic lesions and they can also arise as intermediates of DNA repair activity. Unrepaired DNA damage can lead to genomic instability, which can massively compromise the health status of organisms. Therefore it is important to measure and quantify DNA damage and its repair.
\end{abstract}

Results: We have previously published an automated method for measuring DNA strand breaks based on fluorimetric detection of alkaline DNA unwinding [1], and here we present a mathematical model of the FADU assay, which enables to an analytic expression for the relation between measured fluorescence and the number of strand breaks.

Conclusions: Assessment of the formation and also the repair of DNA strand breaks is a crucial functional parameter to investigate genotoxicity in living cells. A reliable and convenient method to quantify DNA strand breakage is therefore of significant importance for a wide variety of scientific fields, e.g. toxicology, pharmacology, epidemiology and medical sciences.

Keywords: FADU, DNA repair, DNA strand breaks, Mathematical model

\section{Background}

The fact that the DNA denatures ("unwinds") under alkaline conditions has been used to establish FADU assay for measuring DNA strand breaks [2]. This method is based on the limited denaturation of the DNA under precisely controlled conditions of $\mathrm{pH}$, temperature and time. Under these conditions only chromosome ends plus "open sites" in the DNA serve as starting points for the unwinding, which proceeds bidirectionally from internal damage sites. After stopping the unwinding process at a specified time, a dye is added that interacts with the remaining double stranded DNA and during contact with DNA emits a fluorescence signal whose intensity is inversely related to the number of DNA strand breaks originally present: the less fluorescence signal, the more DNA strand breaks.

\footnotetext{
* Correspondence: maria.moreno-villanueva@uni-konstanz.de

${ }^{2}$ Molecular Toxicology Group, Department of Biology, University of Konstanz, D-78457 Konstanz, Germany

Full list of author information is available at the end of the article
}

When X-radiation is applied to living cells, the number of strand breaks is known to increase linearly with the dose applied [3,4]. However, the fluorescence signal intensity depends on the dose in a nonlinear fashion and displays saturation. Previously, the percentage of double stranded DNA, remaining after the alkaline unwinding, relative to undenatured control or more precisely the decadic logarithm of the intensity ratio with and without unwinding has been used for that purpose, but this does not fully result in a linear function with radiation dose. In this article, we present a mathematical model that describes the effect of DNA unwinding on the resulting strength of the fluorescence signal and thereby captures the non-linear relationship more precisely.

The main assumptions of the model are large cell numbers, homogeneously distributed damage sites, and small variation of cell parameters like initial damage, susceptibility to applied damage, and potential unwinding 
distance from the site of damage. The resulting formula relates the relative fluorescence intensity to the applied $\mathrm{X}$-ray dose and fits well with experiments. To obtain this result, it suffices to use elementary arguments from probability theory. In particular, a detailed biochemical model of the unwinding process as in [5-8] is not required.

\section{Results}

We consider $N$ cells of identical type, which are subjected to a controlled DNA damage by a specified dose of $\mathrm{X}$-radiation. After the treatment, the DNA in cell $i$ exhibits a certain number of (single or double) strand breaks. We denote this damage $D_{i}$ and split it into a pre-existing, initial contribution $D_{i}^{0}$ and an induced damage $D_{i}^{x}=\beta_{i} d$ which is proportional to the applied dose $d$. In mathematical terms, the FADU assay assigns to each $D_{i}$ a corresponding fluorescence signal $F_{i}$ of the cell and the total fluorescence $F=\sum_{i=1}^{N} F_{i}$ of all cells is measured. Ideally, the fluorescence $F_{i}$ is related, after completion of the FADU assay, to the length of single and double stranded DNA in cell $i$ according to

$$
F_{i}=c\left(B\left(1-L_{i}\right)+L_{i}\right)
$$

Here $L_{i}$ is the relative length of double stranded DNA in the cell so that $1-L_{i}$ is the relative length of DNA, which has been unwound. In particular, $c$ is the fluorescence signal that is obtained in the case of no unwinding $L_{i}=1$, and $c B$ the one after complete unwinding $L_{i}=0$ (we call $B$ the relative background fluorescence). Normalizing the total fluorescence $F$ with the one in the case of no unwinding $F^{0}=N c$, we obtain

$$
I=\frac{F}{F^{0}}=\frac{1}{N} \sum_{i=1}^{N} B\left(1-L_{i}\right)+L_{i}=B+(1-B) \frac{1}{N} \sum_{i=1}^{N} L_{i}
$$

In other words, the relative fluorescence intensity $I$ depends on the average relative length of double stranded DNA and the relative background fluorescence $B$ under total unwinding. Later, the fact that $N$ is large allows us to apply the mathematical law of large numbers to safely replace the average by an expected value.

It remains to model the relative length $L_{i}$ of double stranded DNA after the unwinding process and its relation to the damage $D_{i}$. Since we assume that the process happens identically and independently in each cell, we consider a generic situation and drop the cell index $i$ in the following discussion.

Our basic assumption is that the break points $x_{k}$ are uniformly distributed along the DNA strands and that the DNA unwinds a distance $\delta$ to the left and to the right of $x_{k}$ unless there is interference with the unwinding process going on at a neighboring break.
Since unwinding also starts at the ends of the chromosomes, it is useful to unify the description by hypothetically stringing the various chromosomes together. As far as unwinding is concerned, this does not change the result as long as the points which mark the relative stringing positions are added to the list of break points. This trick leads to an elegant description of the unwinding process: We start from $m$ internal strand breaking points $0<\mathrm{z}_{1}<\cdots<\mathrm{z}_{m}<1$ and associate to each $z_{k}$ the two neighboring points $z_{k}^{ \pm}=z_{k} \pm \delta$ that mark the potential unwinding interval $\left[z_{k}^{-}, z_{k}^{+}\right]$which is also the actual unwinding if there is no interference with neighboring intervals. Obviously, such an interference appears between $z_{k}$ and $z_{k+1}$ if and only if $z_{k}^{+}>z_{k+1}^{-}$. Altogether, the contribution to the relative length $L$ of double stranded DNA between $z_{k}$ and $z_{k+1}$ is $\left(z_{k+1}^{-}-z_{k}^{+}\right)_{+}$where $u_{+}=\max (u, 0)$ denotes the positive part of a number $u$. Introducing the auxiliary points $z_{0}=0, z_{m+1}=1$ and summing up all the contributions, we obtain

$$
L=\sum_{k=0}^{m}\left(z_{k+1}^{-}-z_{k}^{+}\right)_{+}=\sum_{k=0}^{m}\left(z_{k+1}-z_{k}-2 \delta\right)_{+}=\sum_{k=0}^{m}\left(\Delta_{k}-2 \delta\right)_{+},
$$

where $\Delta_{k}=z_{k+1}-z_{k}$ is the distance between consecutive break points. For $m$ we use the splitting $m+1=D^{0}+D^{x}$ into the number of breaks $D^{x}=\beta d$ due to X-ray damage and the number $D^{0}$ accounting for breaks at zero damage, resulting from normal cell metabolism. Note that in our model, $D^{0}$ is at least the number of chromosomes because we have added their terminal points as artificial breaks. Due to the stochastic nature of the damaging process, the cell metabolism and the unwinding, the number of strand breaks per dose $\beta$, the zero dose value $D^{0}$ and the unwinding distance $\delta$ should be considered variables whose value may vary randomly from cell to cell.

To estimate the resulting distribution of the relative fluorescence signal $f$ with some manageable expression it seems worthwhile to simplify the model further, even if it requires additional assumptions. For example, the situation simplifies a lot if we assume that all $z_{1}, \ldots, z_{m}$ are independently and uniformly distributed in the interval $[0,1]$, which means that the particular role of the original terminal chromosome points is dropped. Since in a normal diploid human cell, the number of chromosomes is only 46, this may be acceptable as long as $m$ is comparably large.

According to [9], the uniform distribution of the points $z_{k}$ implies that the distances $\Delta_{k}=z_{k+1}-z_{k}, k=0, \ldots, m$ between the points are independent and identically distributed like $\Delta^{(m)}=1-\sqrt[m]{U}$ where $U$ is uniformly distributed on $[0,1]$. In other words, $\Delta^{(m)}$ has a probability density $m(1-x)^{m-1}$ for $x \in[0,1]$. This result allows us to compute the conditional expectation 


$$
E(L \mid m)=\sum_{k=0}^{m} E\left(\Delta_{k}-2 \delta\right)_{+}=(m+1) E\left(\Delta^{(m)}-2 \delta\right)_{+} .
$$

For a given value $\delta$ we have in the case $2 \delta<1$

$$
E\left(\Delta^{(m)}-2 \delta\right)_{+}=\int_{2 \delta}^{1}(x-2 \delta) m(1-x)^{m-1} d x=\frac{(1-2 \delta)^{m+1}}{m+1},
$$

while $E\left(\Delta^{(m)}-2 \delta\right)_{+}=0$ when $2 \delta \geq 1$. Combining this result with the previous formula, we find the conditional expectation

$$
E\left(L \mid D^{0}, \beta, \delta\right)=(1-2 \delta)_{+}^{D^{0}+\beta d} .
$$

In order to compute the unconditional expectation $E(L)$, reasonable assumptions on the probability distributions of $D^{0}, \beta$ and $\delta$ are required. Finally, $E(L)$ depends in a complicated way on the dose $d$, the mean values $\overline{D^{0}}, \bar{\beta}, \bar{\delta}$, the corresponding variances and maybe other parameters of the distributions.

However, since little information on the distributions of $D^{0}, \beta$ and $\delta$ is available, we continue in a different way. We assume that the unconditional expectation $E(L)$ can be approximated with an expression of the form

$$
E(L) \approx\left(1-2 \delta_{e f f}\right)^{D_{e f f}^{0}+\beta_{e f f} d}
$$

which is a reasonable assumption when $D^{0}, \beta$ and $\delta$ have negligible variance among all cells in the assay. Then, the effective values $D_{\text {eff }}^{0}, \beta_{\text {eff }}$ and $\delta_{\text {eff }}$ are close to the mean values $\overline{D^{0}, \bar{\beta}}, \bar{\delta}$.

Replacing the average in the formula for relative intensity $I$ by the approximate expectation, we obtain

$$
I \approx P(d)=B+(1-B)\left(1-2 \delta_{e f f}\right)^{D_{e f f}^{0}+\beta_{e f f} d} .
$$

More compactly, this relationship can be written in the form

$$
P(d)=B+\left(P_{0}-B\right) e^{-\lambda d},
$$

where the relation between the fit parameters $P_{0}, \lambda$ and the model parameters is

$$
P_{0}=B+(1-B)\left(1-2 \delta_{e f f}\right)^{D_{e f f}^{0}}, \quad \lambda=-\beta_{e f f} \ln \left(1-2 \delta_{e f f}\right) .
$$

Once $B, P_{0}, \lambda$ are determined, the equivalent dose value $d$ corresponding to the relative intensity $P$ can easily be determined by inversion

$$
d=-\frac{1}{\lambda} \ln \frac{P-B}{P_{0}-B} .
$$

Unfortunately, an estimate of $D_{\text {eff }}^{0}+\beta_{\text {eff }} d$ which is essentially the total number of strand breaks is not available if only $B, P_{0}, \lambda$ are known. However, a related dose-equivalent value

$$
D_{t o t}=\frac{D_{e f f}^{0}+\beta_{e f f} d}{\beta_{e f f}}
$$

is accessible. In fact, applying the logarithm to $\left(P_{0}-B\right) /$ $(1-B)$ and using the relation for $\lambda$, we find

$$
\frac{D_{e f f}^{0}}{\beta_{e f f}}=-\frac{1}{\lambda} \ln \frac{P_{0}-B}{1-B} .
$$

Together with the expression for the dose value $d$, we obtain a formula that relates $P$ to the value $D_{\text {tot }}$.

$$
D_{t o t}(P)=\frac{D_{e f f}^{0}+\beta_{e f f} d}{\beta_{e f f}}=-\frac{1}{\lambda} \ln \frac{P-B}{1-B}
$$

In order to test our model, we use experimental results from the experiment specified in Material and Methods. Here, three replicates of the relative intensities at seven dose values are available for eleven donors. We denote them $P_{j k l}$ where the index $j=0, \ldots, 6$ labels the X-ray dose, $k=1, \ldots, 11$ represents the donor and $l=1,2,3$ indicates the independent repetitions of the experiment.

While each donor may exhibit different parameters $P_{0 k}, \lambda_{k}$, the background intensity $B$ can be assumed identical in all experiments. To determine the unknown coefficients, we want to minimize the objective function

$$
\sum_{k=1}^{11} \sum_{j=0}^{6} \sum_{l=1}^{3}\left(B+\left(P_{0 k}-B\right) \exp \left(-\lambda_{k} d_{j}\right)-P_{j k l}\right)^{2} \underset{B, \lambda_{k}, P_{0 k}}{\rightarrow} \min
$$

which leads to the same result as the minimization of

$$
z\left(B, \lambda, P_{0}\right)=\sum_{k=1}^{11} \sum_{j=0}^{6}\left(B+\left(P_{0 k}-B\right) \exp \left(-\lambda_{k} d_{j}\right)-\bar{P}_{j k}\right)^{2}
$$

based on the averages

$$
\bar{P}_{j k}=\frac{1}{3} \sum_{l=1}^{3} P_{j k l} .
$$

To quantify the quality of the fit, we check how much of the total sum of squares

$$
S S_{t o t}=\sum_{k=1}^{11} \sum_{j=0}^{6}\left(\bar{P}_{j k}-\bar{P}\right)^{2}, \quad \bar{P}=\frac{1}{77} \sum_{k=1}^{11} \sum_{j=0}^{6} \bar{P}_{j k}
$$

is explained by the fitting which leads to an $R^{2}$-value

$$
R^{2}=1-\frac{S S_{\text {err }}}{S S_{\text {tot }}},
$$

where $S S_{\text {err }}=z\left(B^{*}, \lambda^{*}, P_{0}^{*}\right)$ is the residual in case of the optimal parameters. The optimization is carried out with 
the Levenberg-Marquardt algorithm of the Matlab software package with a resulting value of $R^{2}=0.99$.

Confidence intervals for the fit parameters are computed with the bootstrap method [10] exploiting the information contained in the threefold repetition of each experiment. For each index combination $(j, k, l), N=10000$ bootstrap samples $P_{j k l}^{n}$ are generated by drawing intensities randomly from the values $P_{j k 1}, P_{j k 2}, P_{j k 3}$ with replacement. Then the computation of the optimal parameters is repeated for each $n=1, \ldots, N$ and the resulting distribution is used to construct $95 \%$-confidence intervals.

The resulting value for $B$ is

$$
B=0.122 \pm 0.008 \text {. }
$$

The values $\lambda_{k}, P_{0 k}$ for each donor are reported in Table 1 and the quality of the fit is demonstrated in Figure 1.

\section{Discussion}

DNA strand breaks are one of the most common genotoxic lesions. One of the methods used for measuring DNA strand breaks and their repair is the FADU assay. Under the selected alkaline conditions a large number of base pairs will be unwound to the right and left starting from each DNA "open side". Due to this amplification it is possible to detect very low number of DNA strand breaks. The main advantage of the FADU assay is that it can be performed in a fully automated fashion. However there are few methods that can be performed in a semiautomated version such as the COMET and $\gamma \mathrm{H} 2 \mathrm{AX}$ assays. Even though these techniques provide useful tools for measuring DNA strand breaks in a high-throughput

Table 1 Fit parameters for the relative fluorescence intensity

\begin{tabular}{lll}
\hline Donor & $\boldsymbol{P}_{\mathbf{0}}$ & $\boldsymbol{\lambda}$ \\
\hline 1 & $0.79 \pm 0.01$ & $0.18 \pm 0.02$ \\
2 & $0.74 \pm 0.06$ & $0.24 \pm 0.04$ \\
3 & $0.64 \pm 0.04$ & $0.21 \pm 0.03$ \\
4 & $0.65 \pm 0.02$ & $0.21 \pm 0.02$ \\
5 & $0.51 \pm 0.04$ & $0.20 \pm 0.05$ \\
6 & $0.65 \pm 0.05$ & $0.20 \pm 0.04$ \\
7 & $0.60 \pm 0.03$ & $0.30 \pm 0.04$ \\
8 & $0.63 \pm 0.02$ & $0.25 \pm 0.02$ \\
9 & $0.66 \pm 0.04$ & $0.31 \pm 0.05$ \\
10 & $0.74 \pm 0.03$ & $0.28 \pm 0.02$ \\
11 & $0.66 \pm 0.08$ & $0.18 \pm 0.06$ \\
\hline
\end{tabular}

The zero-dose value $P_{0}$ shows a considerable variation among the donors ranging from 0.5 to 0.8 , which is roughly three times the typical width of a confidence interval. In contrast, the exponential decay parameter has much less variation in the range 0.18 to 0.31 , which amounts only to a single width of the typical confidence intervals. fashion, they have some disadvantages. A comprehensive comparison between these assays has been published before [11]. Briefly, $\gamma \mathrm{H} 2 \mathrm{AX}$ assay is considered a very sensitive method that specifically detects double DNA strand breaks however in some cases the presence of $\gamma \mathrm{H} 2 \mathrm{AX}$ foci in the absence of DNA damage has been demonstrated. The comet assay protocol includes many different steps making difficult the development of a fully automated version. Furthermore these also affect both intra-assay variability and inter-assay reproducibility.

We have previously published a modified and automated version of the FADU assay $[1,11,12]$. Compared with the original FADU assay [2], the automated FADU assay shows comparable sensitivity, yet increased robustness and throughput, and decreased operator time. However a suitable mathematical model, which allows translating the fluorescence intensity in DNA damage increases accuracy. In order to test whether the model is capable of describing the experimentally observed dependence between applied damage and resulting fluorescence intensity, we have measured the DNA strand breaks in peripheral mononuclear blood cells of 11 individuals. It turns out that the model is able to fit the dose-effect relation very accurately.

As shown in Table 1, the resulting model parameters $\lambda$ and $P_{0}$, which describe the cell's susceptibility to DNA damage and the level of endogenous DNA strand breaks respectively, show a certain variation among the individuals which is stronger for the $P_{0}$ value. An individual endogenous level of DNA strand breaks is expected; in general the importance of endogenous DNA damage has been reported before [13]. For example, it is known that DNA double-strand breaks (DSBs) accumulate in senescing human cell cultures and in ageing mice [14]. This is in accordance with our recent data showing that senescing human T lymphocytes have acquired more DNA strand breaks in vivo and an impaired DNA repair capacity in vitro [15]. Additionally, endogenous DNA strand breaks accumulation in human cells has been associated with nutrition [16], cancer [17], diabetes [18,19], Down's syndrome [20], rheumatoid arthritis [21] and psychological stress disorders [22], among others. In other words, differences in the level of endogenous DNA strand breaks might reflect aging processes or health status of individuals.

The susceptibility to DNA damage $\lambda$ also shows variation between individuals. This finding is not surprising and has been described before. Odarigi and colleagues reported an inter-individual variance in chromosomal damage after $x$-irradiation [23]. Since radiation induces free oxygen radical formation, an individual damage response to $\mathrm{x}$-ray might result from the different capacity of cells to scavenge these free radicals.

The investigation of DNA strand breaks and their repair is of great interest to toxicology, pharmacology, 


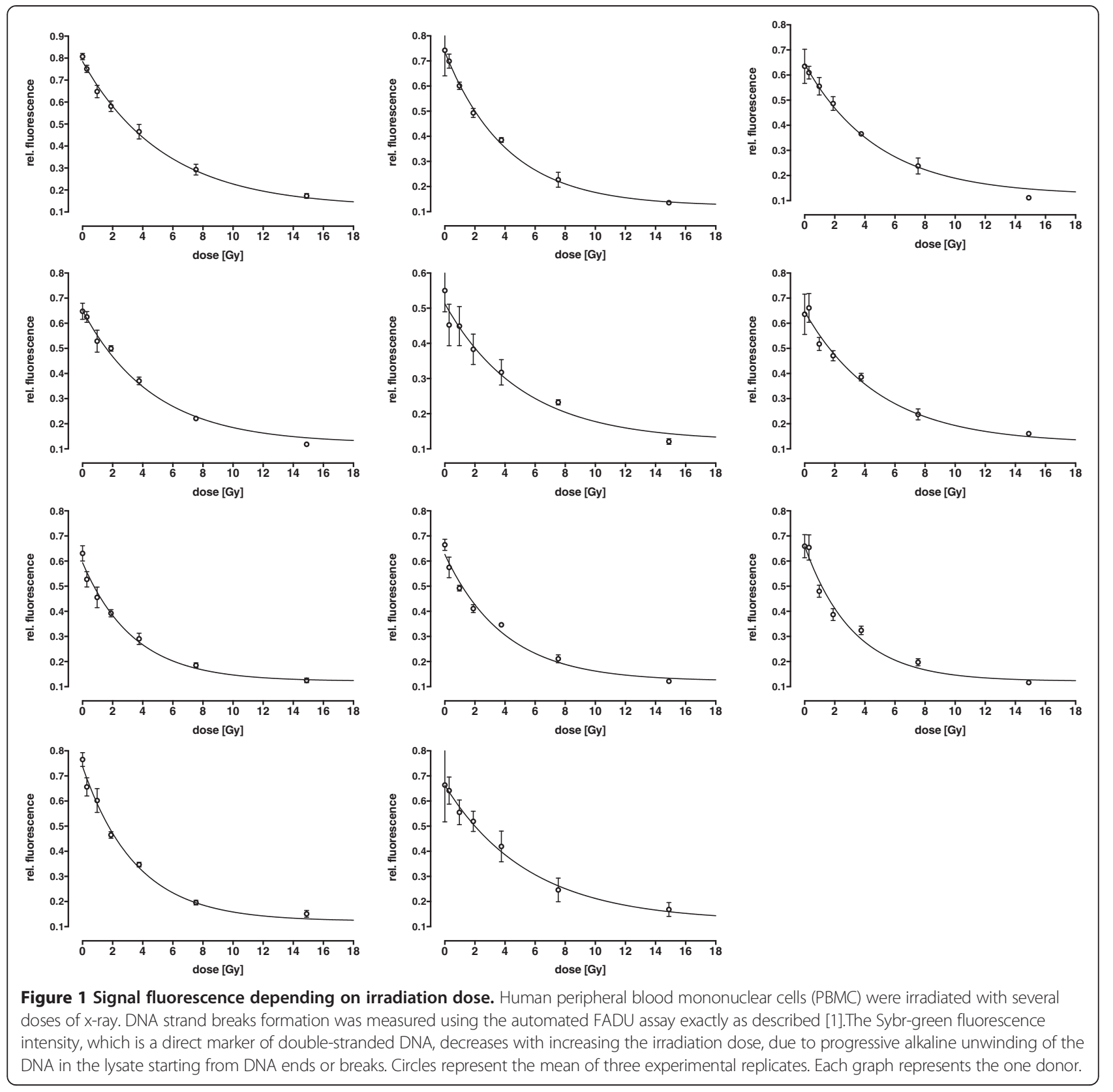

epidemiology and medical sciences at large. In general impaired DNA repair can lead to embryonic lethality, impaired growth, accelerated aging, shortened life span, and increased incidence of a variety of diseases, including a pronounced manifestation of cancer [24-28]. Therefore robust methods for quantification of DNA damage and its repair are necessary.

Finally, in our model, induced DNA strand breaks are assumed to be uniformly distributed. However chromatin state could have an impact on the distribution of DNA strand breaks. Chromatin state differs depending on cell type and cell status (cell cycle phase and/or transcription) therefore it is important to take these aspects into consideration. A mathematical model of DNA unwinding considering the chromatin state would be an interesting task for future research, especially when comparing different cell types or cell status. A more refined model could be based on a probability distribution, which describes the susceptibility to breaking under X-ray exposure along the DNA strand.

\section{Conclusions}

We have applied the above mathematical model for the quantification of DNA strand breaks in irradiated human PBMCs. This model can be also applied to other cell types and other DNA damaging agents as long as the damaging 
agent leads to uniformly distributed DNA strand breaks. Furthermore measuring the residual DNA strand breaks in damaged cells after several periods of time allow us to calculate the rate of repaired DNA strand breaks. Last but not least the amount of endogenous DNA strand breaks provides important information about the individual "cell-damage-status" and fluorescence signal after induced damage serves as biomarker of cell sensitivity to DNA strand breaks inducers, therefore an accurate model for DNA breakage quantification could be used for diagnostic and therapy purposes towards a personalized medicine, e.g. in cancer therapy.

\section{Methods}

Selection of volunteers was carried out in accordance with the Declaration of Helsinki and ethical approval was obtained from the Ethics Committee of University of Constance. A signed Informed Consent was obtained from each subject. Blood from healthy volunteers aged 23-55 was collected in sodium citrat tubes (Sarstedt, Nümbrecht, Germany). The automated FADU assay has previously been described in detail [1]. Briefly, peripheral blood mononuclear cells (PBMC) were isolated by Biocoll (Biochrom, Berlin, Germany) density gradient centrifugation. Cells were counted using a cell counting device (Casy counter), pelleted (10 min, $200 \mathrm{~g}$ ), and resuspended in isotonic buffer $(0.25 \mathrm{M}$ meso-inositol; $10 \mathrm{mM}$ sodium phosphate, pH 7.4; 1 mM magnesium chloride) or RPMI1640 medium (Invitrogen) containing $100 \mathrm{U} / \mathrm{ml}$ penicillin (Invitrogen), $100 \mathrm{mg} / \mathrm{ml}$ streptomycin (Invitrogen) and $10 \%$ fetal calf serum (FCS) at $4 \times 10^{6}$ cells per ml. In order to induce DNA strand breaks several aliquots of $100 \mu \mathrm{l}$ cell suspension were irradiated on ice at a dose rate of $1.9 \mathrm{~Gy} / \mathrm{min}$ using an X-ray generator (CHF Müller, Hamburg, Germany, $70 \mathrm{keV}, 1 \mathrm{~mm}$ Al-filter). Qualitative behaviour has been modelled, but it still depends on few quantitative parameters, which where determined based on the results obtained from unwinding experiments after various $(0.3,1,1.9,3.8,7.5$ and $14.9 \mathrm{~Gy})$ irradiation doses (Figure 1).

The detection of DNA strand breaks is based on progressive DNA unwinding (denaturation) under highly controlled conditions of alkaline $\mathrm{pH}$, time and temperature. The starting points for the unwinding process are DNA "open sites" like replication forks or chromosome ends, but also DNA strand breaks. For monitoring DNA damage a commercially available fluorescent dye (SYBR Green ${ }^{\circ}$ ) is used as marker for double stranded DNA, and a decrease in the fluorescence intensity of SYBR Green indicates an increase of DNA unwinding and consequently a greater number of DNA strand breaks.

$\mathrm{T}$ and $\mathrm{P}_{0}$ are controls to be run in parallel with the experimentally treated cells. In P0 samples alkaline unwinding is allowed and represents the DNA strand breaks under physiological conditions (i.e. without exogenous DNA damage). In T samples the neutralisation follows the lysis and therefore unwinding cannot take place; Tsamples provide a measure of total DNA content and yield a fluorescence signal defined as $100 \% . \mathrm{P}_{\mathrm{x}}$ samples $\left(\mathrm{P}_{1}, \mathrm{P}_{2}, \mathrm{P}_{3}\right.$, $\left.\mathrm{P}_{4} \ldots \mathrm{P}_{\mathrm{x}}\right)$ are the different extent of damage to be measured.

\section{Competing interests}

The authors declare that they have no competing interests.

\section{Authors' contributions}

The work presented here was carried out in collaboration between all authors. MMV defined the research theme and designed methods and experiments. JS carried out the laboratory experiments and analyzed the data. TS co-worked on data interpretation and discussed analyses. MJ developed the mathematical model and carried out the parameter fitting. AB co-designed experiments, discussed analyses, interpretation, and presentation. MMV and MJ wrote the paper. All authors have contributed to, seen and approved the manuscript.

\section{Acknowledgements}

This work was supported by the European Union FP7 Health research, grant number HEALTH-F4-2008-200880 [MARK-AGE]. The authors would like to acknowledge Ms. Gudrun von Scheven for organizing the blood collection.

\section{Author details}

${ }^{1}$ Numerics group, Department of Mathematics and Statistics, Universität Konstanz, D-78457 Konstanz, Germany. ${ }^{2}$ Molecular Toxicology Group, Department of Biology, University of Konstanz, D-78457 Konstanz, Germany.

Received: 8 May 2014 Accepted: 19 August 2014

Published online: 09 September 2014

\section{References}

1. Moreno-Villanueva M, Pfeiffer R, Sindlinger T, Leake A, Müller M, Kirkwood T, Bürkle A: A modified and automated version of the 'Fluorimetric Detection of Alkaline DNA Unwinding' method to quantify formation and repair of DNA strand breaks. BMC Biotechnol 2009, 9:39.

2. Birnboim HC, Jevcak JJ: Fluorometric method for rapid detection of DNA strand breaks in human white blood cells produced by low doses of radiation. Cancer Res 1981, 41:1889-1892.

3. Wood DK, Weingeist DM, Bhatia SN, Engelward BP: Single cell trapping and DNA damage analysis using microwell arrays. PNAS 2010, 1;107(22):10008-10013

4. Olive PL, Banáth JP: The comet assay: a method to measure DNA damage in individual cells. Nat Protoc 2006, 1(1):23-29.

5. Gaver DP, Jacobs PA, Carpenter RL, Burkhart JG: A mathematical model for intracellular effects of toxins on DNA adduction and repair. Bull Math Biol 1997, 59(1):89-106.

6. Sokhansanj BA, Rodrigue GR, Fitch JP, Wilson DM: A quantitative model of human DNA base excision repair. I. Mechanistic insights. Nucleic Acids Res 2002, 30(8):1817-1825.

7. Kesseler KJ, Kaufmann WK, Reardon JT, Elston TC, Sancar A: A mathematical model for human nucleotide excision repair: damage recognition by random order assembly and kinetic proofreading. J Theor Biol 2007, 249(2):361-375.

8. Crooke PS, Parl FF: A mathematical model for DNA damage and repair. J Nucleic Acids 2010, 2010:352603.

9. Devroye L: Non-Uniform Random Variate Generation. New York: Springer Verlag; 1987.

10. Efron B, Tibshirani RJ: An Introduction to the Bootstrap. In Monographs on Statistics and Applied Probability 57. Chapman\&Hall/CRC; 1994.

11. Moreno-Villanueva M, Bürkle A: High-throughput Assays to Quantify the Formation of DNA Strand Breaks. In High Throughput Screening Methods in Toxicity Testing. Edited by Steinberg P. Hoboken, NJ, USA: John Wiley \& Sons, Inc; 2013:285-294.

12. Moreno-Villanueva M, Eltze T, Dressler D, Bernhardt J, Hirsch C, Wick P von Scheven G, Lex K, Bürkle A: The automated FADU-assay, a potential high-throughput in vitro method for early screening of DNA breakage. ALTEX 2011, 28(4):295-303. 
13. De Bont $R$, van Larebeke $N$ : Endogenous DNA damage in humans: a review of quantitative data. Mutagenesis 2004, 19(3):169-185.

14. Sedelnikova OA, Horikawa I, Zimonjic DB, Popescu NC, Bonner WM, Barrett JC: Senescing human cells and ageing mice accumulate DNA lesions with unrepairable double-strand breaks. Nat Cell Biol 2004, 6(2):168-170.

15. Brunner S, Herndler-Brandstetter D, Arnold CR, Wiegers GJ, Villunger A, Hackl M, Grillari J, Moreno-Villanueva M, Bürkle A, Grubeck-Loebenstein B: Upregulation of miR-24 is associated with a decreased DNA damage response upon etoposide treatment in highly differentiated CD8(+) T cells sensitizing them to apoptotic cell death. Aging Cell 2012, 11(4):579-587.

16. Krajcovicová-Kudlácková M, Dusinská M: Oxidative DNA damage in relation to nutrition. Neoplasma 2004, 51(1):30-33.

17. Vilenchik MM, Knudson AG: Endogenous DNA double-strand breaks: production, fidelity of repair, and induction of cancer. Proc Natl Acad Sci 2003, 100(22):12871-12876.

18. Lorenzi M, Montisano DF, Toledo S, Wong HC: Increased single strand breaks in DNA of lymphocytes from diabetic subjects. J Clin Invest 1987, 79(2):653-656.

19. Blasiak J, Arabski M, Krupa R, Wozniak K, Zadrozny M, Kasznicki J, Zurawska M, Drzewoski J: DNA damage and repair in type 2 diabetes mellitus. Mutat Res 2004, 554(1-2):297-304.

20. Zana M, Szécsényi A, Czibula A, Bjelik A, Juhász A, Rimanóczy A, Szabó K, Vetró A, Szucs P, Várkonyi A, Pákáski M, Boda K, Raskó I, Janka Z, Kálmán J: Age-dependent oxidative stress-induced DNA damage in down's lymphocytes. Biochem Biophys Res Commun 2006, 345(2):726-733. Epub 2006 May 5.

21. Bhusate LL, Herbert KE, Scott DL, Perrett D: Increased DNA strand breaks in mononuclear cells from patients with rheumatoid arthritis. Ann Rheum Dis 1992, 51(1):8-12.

22. Morath J, Moreno-Villanueva M, Gilava H, Kolassa S, Ruf-Leuschner M, Schauer M, Elbert T, Bürkle A, Kolassa I-T: Effects of psychotherapy on DNA strand break accumulation originating from traumatic stress. Psychother Psychosom 2014, 83(5):289-297.

23. Odagiri $Y$, Uchida H, Shibazaki S: Interindividual variation in cytogenetic response to X-ray and colchicine measured with the cytokinesis-block micronucleus assay. Mutat Res 1997, 381(1):1-13.

24. Hakem R: DNA-damage repair; the good, the bad, and the ugly. EMBO J 2008, 27:589-605.

25. Diderich $\mathrm{K}$, Alanazi M, Hoeijmakers JH: Premature aging and cancer in nucleotide excision repair-disorders. DNA Repair 2011, 10(7):772-780.

26. Keimling $M$, Deniz M, Varga D, Stahl A, Schrezenmeier $H$, Kreienberg $R$, Hoffmann I, König J, Wiesmüller L: The power of DNA double-strand break (DSB) repair testing to predict breast cancer susceptibility. FASEB J 2012, 26(5):2094-2104

27. Varga $D$, Deniz $M$, Schwentner $L$, Wiesmüller L: Ovarian cancer: in search of better marker systems based on DNA repair defects. Int J Mol Sci 2013, 14(1):640-673.

28. Toulany M, Rodemann HP: Membrane receptor signaling and control of DNA repair after exposure to ionizing radiation. Nuklearmedizin 2010, 2010(49 Suppl 1):S26-S30.

doi:10.1186/s13628-014-0009-z

Cite this article as: Junk et al: Mathematical modelling of the automated FADU assay for the quantification of DNA strand breaks and their repair in human peripheral mononuclear blood cells. BMC Biophysics 2014 7:9.

\section{Submit your next manuscript to BioMed Central and take full advantage of:}

- Convenient online submission

- Thorough peer review

- No space constraints or color figure charges

- Immediate publication on acceptance

- Inclusion in PubMed, CAS, Scopus and Google Scholar

- Research which is freely available for redistribution

Submit your manuscript at www.biomedcentral.com/submit
C Biomed Central 